\title{
Medulloblastoma with an Equivocal Intracerebral Metastasis at Diagnosis
}

\author{
Philip D. Poorvu ${ }^{1 *}$, Gita Suneja ${ }^{1}$, Christine E. Hill-Kayser ${ }^{1}, K^{\prime}$ aruna V. Shekdar ${ }^{2}$ and Giulio J. D’Angio ${ }^{1}$ \\ ${ }^{1}$ Department of Radiation Oncology, University of Pennsylvania, Philadelphia, PA, USA \\ ${ }^{2}$ Department of Radiology, Children's Hospital of Philadelphia, Philadelphia, PA, USA
}

*Corresponding author: Philip D. Poorvu, Brigham and Women's Hospital, 75 Francis Street, Boston, MA 02115, USA, Tel: 617-293-8863; Fax: 617-975-0985; E-mail: ppoorvu@partners.org

Received date: Mar 06, 2014, Accepted date: May 05, 2014, Publication date: May 09, 2014

Copyright: @ 2014 Poorvu PD et al. This is an open-access article distributed under the terms of the Creative Commons Attribution License, which permits unrestricted use, distribution, and reproduction in any medium, provided the original author and source are credited.

\begin{abstract}
Though medulloblastoma frequently involves the leptomeninges at diagnosis, distant metastases are rare on initial presentation. We present a 15-year-old patient with medulloblastoma and equivocal radiographic evidence of an intracerebral metastasis at the time of diagnosis.
\end{abstract}

Keywords: Medulloblastoma; Metastasis; Intracerebral

\section{Case History}

A 15-year-old female presented in April 2011 with a two-year history of intermittent headache, frequent vomiting, and decline in school performance. Her headaches worsened significantly the month prior to presentation with new onset of photophobia and daily vomiting. A computed tomography (CT) scan of the head revealed a mass of the posterior fossa with secondary obstructive hydrocephalus. Magnetic resonance imaging (MRI) revealed that the mass extended through the region of the lateral recess of the fourth ventricle on the left into the cerebellar pontine angle cistern (Figure 1). It compressed the fourth ventricle and appeared to involve the brainstem and cerebellar regions. Two non-enhancing foci of signal abnormality showing restricted diffusion were also noted. One was located in the right parietal lobe and the second involved the medial left temporal lobe near the tentorial notch. The lesion affecting the medial left temporal lobe was interpreted to be a focus of ischemia/edema related to slight transtentorial herniation secondary to hydrocephalus. The lesion in the right parietal lobe, on the other hand, was thought to represent either a leptomeningeal metastatic deposit via cerebrospinal fluid (CSF) seeding or a parenchymal metastasis.

The patient was transferred to our institution and, on the following day, underwent resection of the tumors of the fourth ventricle and the cerebellopontine angle. Pathology was consistent with nodular desmoplastic medulloblastoma, WHO grade IV. Spinal MRI revealed leptomeningeal carcinomatosis. Cytology from lumbar puncture and bone marrow biopsy were negative for malignant cells. Based on radiographic findings alone, the index of suspicion for a parenchymal mass was high and final staging was medulloblastoma M3, with parenchymal brain metastasis and leptomeningeal carcinomatosis.

The patient was treated with craniospinal radiation to $3600 \mathrm{cGy}$, with boosts to the spine to $3960 \mathrm{cGy}$ and to the posterior fossa and parietal metastasis to $5580 \mathrm{cGy}$, in accordance with the Children's Oncology Group ACNS0332 protocol. She received concurrent weekly carboplatin and vincristine. Her radiation therapy course was complicated by a grade 3 skin reaction (moist desquamation) in her lower back, not requiring treatment interruption.

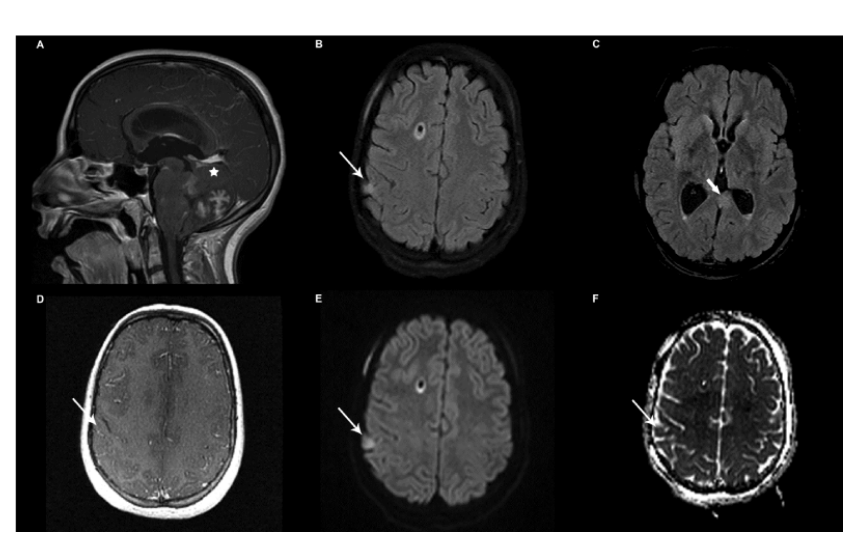

Figure 1: Images from the MRI study at presentation showing the enhancing posterior fossa medulloblastoma (A- white asterisk). Axial FLAIR images demonstrating the hyperintense right parietal lesion considered a parenchymal metastatic deposit (B-thin white arrow) and left medial temporal lesion (C-thick white arrow). No enhancement is seen in the right parietal lesion (D-thin white arrow). $\mathrm{B}=1000$ diffusion image (E) and corresponding ADC map (F) demonstrating restricted diffusion in the right parietal lesion (thin white arrow).

Five weeks following radiation therapy, the patient underwent repeat MRI imaging of the entire central nervous system (CNS), which revealed improved signal abnormality in the left cerebellopontine angle region with no definite tumor seen and resolution of the signal abnormality of the high right parietal lesion (Figure 2). There were persistent spinal leptomeningeal metastases. MRI imaging of the brain and entire spine was repeated four months later and revealed no evidence of residual or recurrent disease. The patient received adjuvant chemotherapy, and nearly 3 years after completing treatment, she remains without evidence of disease. Following treatment, she underwent rehabilitation for residual diplopia, ataxia, and left sided weakness and subsequent neurocognitive testing and audiometry have been normal. To date, she has had no late sequelae of radiation or chemotherapy. 
Citation: Poorvu PD, Suneja G, Kayser CEH, Shekdar KV, Angio GJD (2014) Medulloblastoma with an Equivocal Intracerebral Metastasis at Diagnosis. J Nucl Med Radiat Ther 5: 172. doi:10.4172/2155-9619.1000172

Page 2 of 3

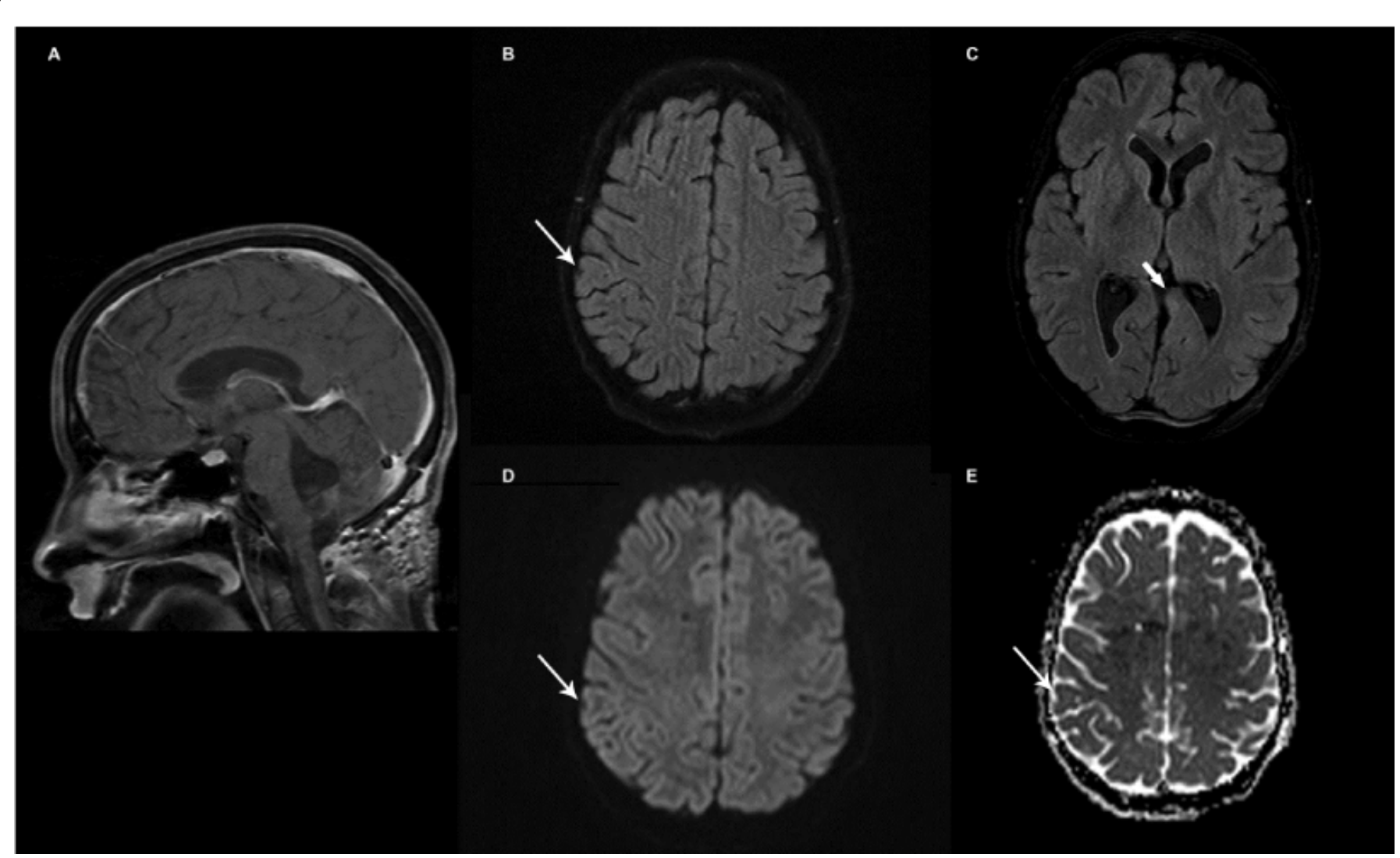

Figure 2: Images from follow-up MRI study following surgical resection, chemotherapy and after completion of radiation therapy showing changes of surgical resection of posterior fossa mass (A). Axial FLAIR images showing resolution of the right parietal lesion (B-thin white arrow) and decreased conspicuity of the left medial temporal lesion (C-thick white arrow). No evidence of restricted diffusion in the location of the previously seen right parietal lesion on $\mathrm{B}=1000$ diffusion image (D) and ADC map (E) consistent with resolution.

\section{Discussion}

Medulloblastoma accounts for $20 \%$ of pediatric brain tumors. Subarachnoid spread within the CNS, both in the spine and supratentorial brain, is common. Up to $30 \%$ of cases present with leptomeningeal carcinomatosis at diagnosis [1,2]. This spread is thought to occur via the CSF. Alternatively, metastasis outside of the CNS, to the lymph nodes, bones, or viscera, typically occurs after surgical intervention [3]. The proposed mechanism of spread is ingrowth of lymphatic and/or vascular channels after transgression of the leptomeninges [3]. Though rare, cases of medulloblastoma with suprasellar metastases at diagnosis have also been reported [4,5].

Cases of medulloblastoma with intracerebral metastases at the time of diagnosis are rare. One reported case was a 6-year-old boy with medulloblastoma of the fourth ventricle and leptomeningeal dissemination who died immediately following surgical intervention. He was found to have an occipital lobe metastasis on autopsy [6]. The occipital lobe mass was histologically nearly identical to the primary tumor and was confirmed to be metastatic medulloblastoma [6]. Pathologic confirmation of the metastasis was not obtained in our patient. This was due to concern of subjecting her to a second surgical resection, particularly given her neurologic deficits following her first surgery. The inability to obtain pathologic confirmation of intracerebral metastasis posed a diagnostic dilemma and therapeutic challenge. Treating the presumed metastasis with high doses of radiation is not without consequence, as the long-term side effects of high dose radiation in the pediatric brain include neurologic, endocrine, and cognitive deficits, as well as secondary malignancies $[7,8]$. Her images were reviewed in detail in a multidisciplinary tumor board, where the consensus opinion was that the index of suspicion for a parenchymal metastasis was sufficiently high to consider her disease M3. This diagnosis was based on three key radiographic features. First, the lesion was isolated with no other evidence of leptomeningeal involvement within the brain and was remote from the area of subarachnoid spread within the spine. Additionally, the mass was nonenhancing while the spinal leptomeningeal metastases were enhancing. Furthermore, the lesion demonstrated restricted diffusion, similar to the posterior fossa primary medulloblastoma.

In this child, the finding of a presumed parenchymal metastasis altered the treatment plan. The patient received a boost dose to both the posterior fossa and the parietal metastasis, yielding a complete response of the deposit. There are short and long-term treatmentassociated side effects after high-dose radiation therapy [8-11]. Acute toxicities include fatigue, headache, insomnia, anorexia, nausea, vomiting, alopecia, and dermatitis, and these may adversely affect quality of life. Late toxicities, such as neurologic, endocrine, and cognitive deficits, can affect quality of life and, in some cases, mortality. The benefit of treating the metastasis to a definitive dose to 
Citation: Poorvu PD, Suneja G, Kayser CEH, Shekdar KV, Angio GJD (2014) Medulloblastoma with an Equivocal Intracerebral Metastasis at Diagnosis. J Nucl Med Radiat Ther 5: 172. doi:10.4172/2155-9619.1000172

Page 3 of 3

achieve local control, however, outweighed the potential long-term toxicity. While pathologic confirmation of the first presentation of metastatic disease may be optimal, it is not always feasible or safe. In cases with a rare presentation of metastatic disease, where pathologic confirmation cannot be safely obtained, reliance on the currently available modern imaging modalities and discussion in a multidisciplinary setting are of critical importance.

\section{Conclusion}

The patient described here is a child with medulloblastoma and presumed intracerebral metastasis at diagnosis. Pathologic confirmation could not be obtained, and we have detailed our diagnostic approach and treatment paradigm.

\section{References}

1. Massimino M, Giangaspero F, Garrè ML, Gandola L, Poggi G, et al (2011) Childhood medulloblastoma. Crit Rev Oncol Hematol 79: 65-83.

2. Packer RJ, Cogen P, Vezina G, Rorke LB (1999) Medulloblastoma: clinical and biologic aspects. Neuro Oncol 1: 232-250.

3. Gyepes MT, D'angio GJ (1966) Extracranial metastases from central nervous system tumors in children and adolescents. Radiology 87: 55-63.
4. Gupta S, Pant MC, Husain N, Sundar S (2011) Metastatic medulloblastoma at diagnosis. J Cancer Res Ther 7: 203-204.

5. Helton KJ, Gajjar A, Hill DA, Boop FA, Kun LE, et al. (2002) Medulloblastoma metastatic to the suprasellar region at diagnosis: a report of six cases with clinicopathologic correlation. Pediatr Neurosurg 37: 111-117.

6. ROSENBERG AW (1957) Intracerebral metastasis of a medulloblastoma: report of case. Bull Los Angel Neuro Soc 22: 36-39.

7. Gunderson LL, Tepper JE (2011) Clinical Radiation Oncology, (3rd edn). Saunders, Philadelphia, PA.

8. Mulhern RK, Palmer SL, Merchant TE, Wallace D, Kocak M, et al. (2005) Neurocognitive consequences of risk-adapted therapy for childhood medulloblastoma. J Clin Oncol 23: 5511-5519.

9. Armstrong GT (2010) Long-term survivors of childhood central nervous system malignancies: the experience of the Childhood Cancer Survivor Study. Eur J Paediatr Neurol 14: 298-303.

10. Geenen MM, Cardous-Ubbink MC, Kremer LC, van den Bos C, van der Pal HJ, et al. (2007) Medical assessment of adverse health outcomes in long-term survivors of childhood cancer. JAMA 297: 2705-2715.

11. Bhat SR, Goodwin TL, Burwinkle TM, Lansdale MF, Dahl GV, et al. (2005) Profile of daily life in children with brain tumors: an assessment of health-related quality of life. J Clin Oncol 23: 5493-5500. 